\title{
VEHICLE DETECTION BY USING REAR PARTS AND TRACKING
} SYSTEM

\author{
Yogini Ashokrao Kanhegaonkar ${ }^{1}$, Jagtap Rupali Ramesh ${ }^{2}$ \\ ${ }^{l}$ M.E Student,Electronics and Telecommunication, Annasaheb Dange College of Engineering, Ashta, Maharashtra, \\ India \\ ${ }^{2}$ Asst. Prof., Electronics and Telecommunication, Annasaheb Dange College of Engineering, Ashta, Maharashtra, \\ India
}

\begin{abstract}
Vision of Indian government; of making 100 smart cities, attracts our attention to intelligent transport system. Traffic flow analysis is a part of intelligent transport system. It mainly contains three parts: vehicle detection, classification and vehicle tracking par t. Recently, there are different detection and tracking methods like computer vision based, magnetic frequency wave based etc. With the rapid development of computer vision techniques, visual detection has become increasingly popular in the transportation field. In urban traffic video monitoring systems, traffic congestion is a common scene that causes vehicle occlusion and is a challenge for current vehicle detection methods. In practical traffic scenarios, occlusion between vehicles often occurs; therefore, it is unreasonable to treat the vehicle as a whole. To overcome this problem we can use part based detection model.

In our system the vehicle is treated as an object composed of multiple salient parts, including the license plate and rear lamps. These parts are localized using their distinctive color, texture, and region feature. Furthermore, the detected parts are treated as graph nodes to construct a probabilistic graph using a Markov random field model. After that, the marginal posterior of each part is inferred using loopy belief propagation to get final vehicle detection. Finally, the vehicles' trajectories are estimated using a Kalman filter and a tracking-based detection technique is realized. This method we can use in daytime as well as night time and in any bad weather condition.
\end{abstract}

Keywords vehicle detection, kalman filter, Markov model, tracking, rear lamps

\section{INTRODUCTION}

\subsection{Traffic Surveillance}

The urban as well as the national road networks have increased in large number for the past thirty years and hence, this development in road networks have necessitated some means to monitor and handle the road traffic. The traditional methods for measuring the traffic include sensors or EM microwave detectors and inductive loops. But, they own severe drawbacks like, traffic disruption at the time of installation and repair, huge size, costlier installation and lack of ability to identify the vehicles that are not fast or not permanently ceased. In contrast, the systems that work depending on video have simple installation steps and utilize the already available traffic surveillance infrastructure. Additionally, enhancements can be made without much difficulty and they are so flexible in redesigning the system as well as the system's functionality through just modifying the algorithms that operate the system. With these systems, it is possible to obtain the measurements that are related to the vehicle such as, speed of the vehicle, calculating the vehicle's count, categorizing the vehicle and finding out the events occurring in the traffic ( like heavy congestion, accidents and similar other events). Plenty of systems, which make use of approaches that come under the field of video and image processing, have been developed for detecting vehicles and the other objects.

\subsection{Video Processing on Traffic Surveillance}

One of the hot research themes in computer vision that draws increased attention is the traffic surveillance system. With this system, the vehicles from an image series can be identified and tracked efficiently. Further, it gives clear details about the behavior of the object or the vehicle in the image series and it makes monitoring as well as the management of traffic easier because it is a better replacement for the traditional method involving human operators, who go around monitoring cameras manually. Using computer vision system, the unauthorized activity that has taken place just before or the mistrustful event that has been produced at an earlier time can be monitored and thus, the human operators are supported in making thorough investigation of the happening.

\subsection{Types of Video Surveillance System}

The various kinds of video surveillance system are as follows:

Manual traffic surveillance system

Semi-automatic traffic surveillance system

Fully-automatic traffic surveillance system 


\subsubsection{Manual Traffic Surveillance System}

This system completely involves a human operator for traffic monitoring. The human operator only observes the visual information, which is captured from various cameras. The operator is subjected to increased level of difficulty, since he has to note all the multiple screens. In addition, the operator is prevented from showing more attention towards adverse happenings. This kind of systems can never prove effective in places that are massive and active because the cameras under usage would rise in number that they go beyond the human expert's ability. These systems are more prevalent in almost all the parts of the world.

\subsubsection{Semi-Automatic Traffic Surveillance System}

In these types of systems, the human operator as well as the computer vision is involved. The computer vision algorithm helps in tracking the object, while the human operator performs other functions like, personal identification, categorization and activity recognition. It employs video processing of lower level and the human operator is mostly involved in monitoring and controlling the traffic.

\subsubsection{Fully-Automatic Traffic Surveillance System}

Here, the computer vision performs all the tasks without the support from the human operator. These systems are more intelligent that they are capable of tracking, classifying and recognizing the object under interest[c]. Moreover, in case of mistrustful events, these systems would intelligently identify those events and performs the object's activity recognition procedure. The visual surveillance systems come into play in situations of urban environment such as, monitoring of congestion in the road, vehicle interaction monitoring and identifying the vehicles that does not follow the rules [23].

\subsection{Need of Video Surveillance System}

The number of severe accidents can be cut down with the video surveillance system and henceforth, so many lives could be safeguarded. A most dedicated application of video analytics that has undergone thorough research is the ANPR. In the toll stations of freeways, devoted lanes containing cameras are available and therefore, the registered users can pass the toll station in a slower manner without ceasing [35]. On the other hand, the inner city congestion charge systems (for instance, Stockholm, Sweden; London, U.K., and Singapore) show decreased level of intrusiveness and function on the traffic that pass in a normal way. In almost all the traffic surveillance systems, the desired traffic parameters that include vehicle identification, tracking and classification can be assessed using three important phases. Majority of the methods [25], [26], [27], [28], [29], [30], [31] that are employed in vehicle detection consider the camera to be static and then, the required vehicles can be identified using the image differencing method. In computer vision, tracking can be thought as a serious problem. Surveillance applications are drawing more attention nowadays.

Tracking in computer vision is mainly required to identify and spot a prototype from a series of sequential frames.
Tracking is increasingly used in applications like security, surveillance and automatic processes as well as video processing. Later, varying tracking schemes are to be designed for tracking every single vehicle. As the next step, the features of different vehicles such as length, shape, texture, width, license number and similar other features are extracted to allow the categorization of vehicles. In all the visual surveillance systems, the first step is the detection of object in motion from the video streams [32]. Traffic surveillance systems can effectively support plenty of applications that include congestion analysis, military security, commercial and public security, crowd flux statistics, visual surveillance and person identification, detection of anomalous behavior and many more.

\subsection{On-Road Vehicle Detection and Tracking}

In all nations, accident avoidance and guaranteeing road safety is an important issue. Thus, several national as well as international researches pertaining to this issue has been developed in recent years [16]. On-road vehicle detection, wherein the vehicle is identified from the images that were captured during the motion of the vehicle on road, is one such research topic [21]. The vehicle detection procedure supports the intelligent transport system applications that include driver-assistance systems, self-guided vehicles and automatic parking systems, in addition to ensuring road safety [19].

In prototype vehicles, active sensors like, lasers can be utilized. The disadvantages of using such sensors are high expense, interference with the other electronic devices and decreased resolution level. Therefore, the utilization of passive sensors like, the camera was more beneficial in the subsequent years. Processing visual data that is acquired from the camera seems to be more significant [18]. Despite the fact that the camera is powerful, processing of poor visual information using passive sensors in the vehicle detection procedure may end in poor reliability levels [17]. It is at this juncture, the computer vision algorithms do an essential task.

\subsection{Practical Challenges}

Some of the serious issues that are faced during the vehicle detection and tracking process include the speed, color and size that are owned by different vehicles, the constant variations in the landscape at the sides of the road, illumination changes in accordance with day and night, weather changes and other parameters that are associated with the vehicle [19] [22]. These issues have been made complex further due to very fast urbanization, traffic and the direction towards which the vehicle moves. Therefore, presently, an efficient as well as automatic vehicle detection and tracking system is under demand [20].

Our aim is introduce a robust vehicle detection and tracking system to investigate the potential features for illustrating the multiple components of the vehicle facilitate the vehicle detection and tracking process improve the performance of vehicle detection and tracking results 
And experimentally investigate the vehicle detection and tracking system and to demonstrate its performance

This paper makes use of the association that exists among the location of the rear lamps and the license plate to accomplish the vehicle detection and tracking approach. The Kalman filter has been employed to get the initial prediction of the object in motion. Yet, the vehicle detection and tracking that is achieved at last is performed using the markov model. This markov model produces the output in accordance with the results of Kalman filter and the relationship that is present between the positions of the rear lamps and the license plates.

The objectives of the project are as follows:

- To develop a robust color space model to segregate rear lamps and rear license plate from the moving vehicle

- To detect and estimate the vehicle based on the rear lamps and license plate with remarkable precision

- To develop an user interface to facilitate the execution of the methodology

- To demonstrate the performance of the methodology

- To ensure the practical viability of the methodology

\section{PROPOSED METHODOLOGY}

The proposed vehicle detection and tracking system is comprised of four stages, namely, vehicle rear lamp detection, rear license plate detection, estimating motion object and detecting vehicle. The architecture diagram of the proposed vehicle detection and tracking algorithm is illustrated in Figure 1.

According to Figure 1, the input video is subjected to image sequencing in which the video is defined as sequence of images in standard size. These image sequences are subjected to three stages, namely rear lamp detection, rear license plate detection and motion estimation using kalman filtering method. A markov model is constructed based on the information obtained from rear lamp detection and rear license plate detection. This markov model is subjected to detect and track the vehicle based on the motion estimation results.

\subsection{Rear Lamps Detection}

The rear lamp detection process gets image sequence and undergoes three basic operations such as color space conversion, post processing and detecting. The image sequences are acquired in RGB color space. However, rear lamps cannot be visualized or detected in this color space. Hence, the sequences are converted from RGB color space to a different color space in which the rear lamps can be detected precisely. We have adopted the color space conversion model proposed in [1], which are as follows.

Please note that math equations might need to be reformatted from the original submission for page layout reasons. This includes the possibility that some in-line equations will be made display equations to create better flow in a paragraph. If display equations do not fit in the two-column format, they will also be reformatted. Authors are strongly encouraged to ensure that equations fit in the given column width

$$
I_{R C}^{i}=I_{R}^{i}-\max \left(I_{G}^{i}, I_{B}^{i}\right)-2\left|I_{G}^{i}-I_{B}^{i}\right|: 0 \leq i \leq N-1
$$

where, $I_{R C}^{i}, I_{R}^{i}, I_{G}^{i}$ and $I_{B}^{i}$ refer to the $i^{\text {th }}$ image in rear lamp color space, $\mathrm{R}$ band, $\mathrm{G}$ band and $\mathrm{B}$ band, respectively.

$\bullet \mid$ represents norm function and $N$ refers to the total number of frames or images in the sequence.

In order to improve the precision of the rear lamp detection precision, a post processing step is included. In the post processing step, firstly the contrast of $I_{R C}^{i}$ is improved followed by binarizing the contrast enhanced image. The binarized image is morphologically eroded and hence the rear lamps are obtained in the images as white pixels, whereas the remaining foreground and background objects appear as black pixels.

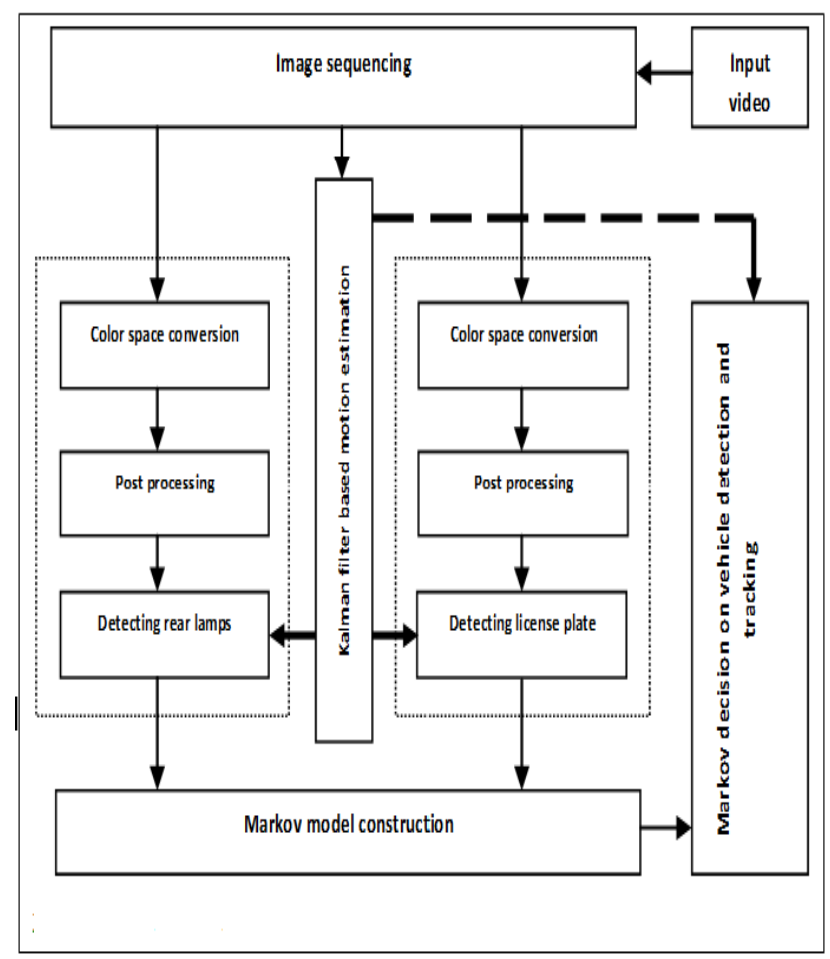

Fig 1 Architecture diagram of the proposed methodology

\subsection{Rear License Plate Detection}

The processes included in detecting the rear license plate of the vehicle are similar to the rear lamp detection processes, except the color space conversion model. According to [1], the color space conversion model that supports rear license plate of the vehicle is given as follows:

$$
I_{L P}^{i}=I_{B}^{i}-\min \left(I_{R}^{i}, I_{G}^{i}\right)
$$


where, $I_{L P}^{i}$ is the $i^{\text {th }}$ image in license plate color space. Similar to rear lamp detection process, the images in license late color space model are subjected to contrast enhancement, binarization and morphological erosion to detect the rear license plate of the vehicle.

\subsection{Motion Estimation using Kalman Filtering}

Motion estimation is the process of determining motion vectors from a sequence of frames. Exploiting kalman filtering process for motion estimation has been reported in the literature since 1990s [2]. It has been referred as one of the promising techniques for effective motion estimation and hence numerous variants of this algorithm have also been reported [3] [4]. Hence, our paper exploits kalman filter to estimate the moving object in the given image sequence. The estimated moving object is used for detecting rear lamps, license plate and also for markov decision.

It is known that in the rear lamp and license plate detection processes, rear lamps and license plate are in white pixels and the other foreground and background objects are in black pixels, respectively. However, it shall not be precise enough to drop all such undesired objects. Based on the kalman filtering output, the misinterpreted rear lamps and license plate are dropped. Similarly, the kalman filtering output is used at the final decision making process also.

\subsection{Markov Model for Detecting/Tracking Vehicle}

The markov model for detecting/ tracking vehicle is constructed based on the relationship between the positions of the left and right rear lamps and the rear license plate. Once the probability of being the vehicle is ensured by the markov model, the final prediction improvement is taken place using the prediction results of kalman filter.

The markov model is constructed based on five interpreted conditions as follows

$$
\begin{aligned}
& \text { Condition (i): } l_{x}>L_{x}^{\text {left }} \\
& \text { Condition (ii): } l_{x}<L_{x}^{\text {right }} \\
& \text { Condition (iii): } L_{x}^{\text {left }}<L_{x}^{\text {right }}
\end{aligned}
$$

$$
\begin{gathered}
\text { Condition (iv): } l_{y}<L_{y}^{\text {left }} \\
\text { Condition (v): }{ }^{l_{y}<L_{y}^{r i g h t}}
\end{gathered}
$$

Based on the aforesaid conditions, emission matrix and the decision model are constructed.

\section{RESULTS AND DISCUSSION}

\subsection{Experimental Results}

The proposed methodology is simulated in MATLAB and experimental investigation is carried out. For a given video, the rear lamps and license plate are detected followed by motion estimation using kalman filter. The image results obtained for the three processes are given in figures 2, 3 and 4 , respectively.

There are multiple localizations of rear lamps and license plates. For instance, left top and right bottom images of figure 2 have more minor centroids. However, these imprecise centroids will be neglected, when the conditions given in Equations (3) - (7) are considered. Similar circumstances occur

in figure 3 also. Figure 4 illustrates the prediction of moving object by kalman filter. These prediction results are highly useful for the markov model to detect and track the vehicles.

\subsection{Error Metric}

The performance of the proposed methodology is investigated using error metrics. The error is determined as the absolute difference between the centroid of the actual vehicle and predicted vehicle. There are 56 frames in the subjected input video and hence we have determined 56 error metrics for all the frames followed by averaging them. Thus obtained mean error is around 11.40 (approx.). The box plot and instantaneous error are illustrated in figures 5 and 6 , respectively.

The box plot in figure 5 shows that the median error metric remains in around 8 (approx.), whereas the minimum and maximum error reach to 0 (approx.) and 32 (approx.), respectively. Few outliers shall go beyond 100 (approx.). More detailed and instantaneous version of the metrics can be visualized through figure 6 .

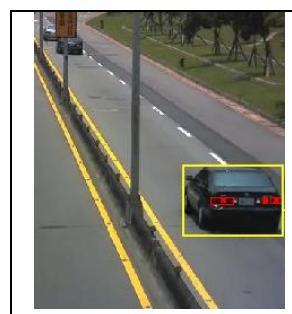

(a)

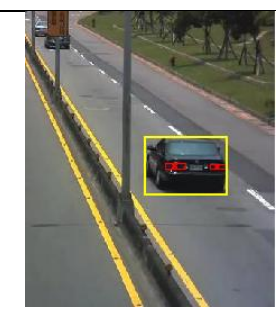

(b)

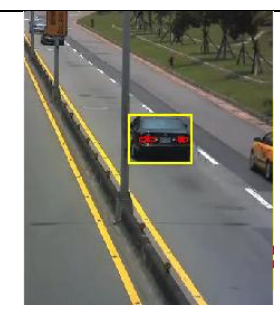

(c)

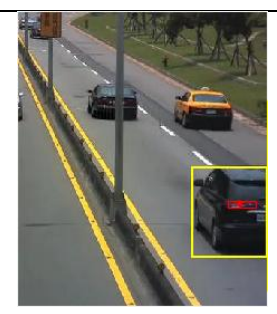

(d)

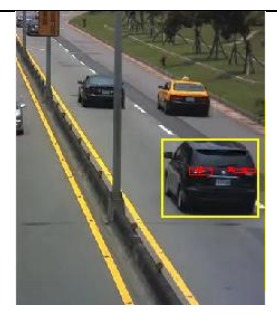

(e)

Fig.2. Detection and tracking of rear lamps for an image sequence. 


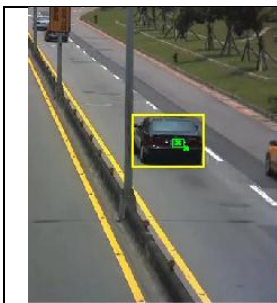

(a)

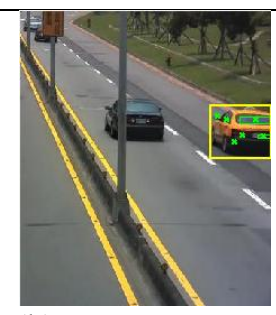

(b)

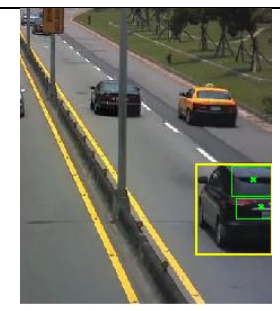

(c)

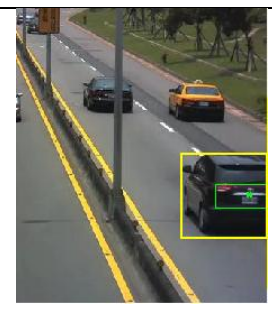

(d)

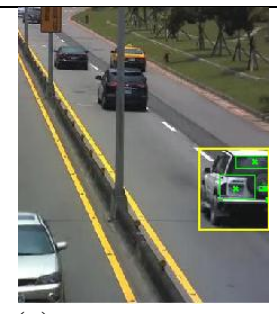

(e)

Fig. 3. Detection and tracking of license plate for an image sequence. Please note that the given sequence is intermediate samples and not temporally interlinked with each other.

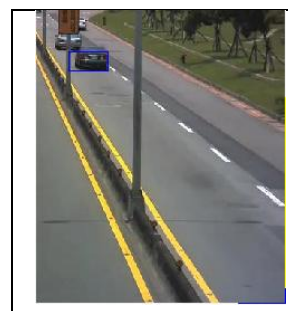

(a)

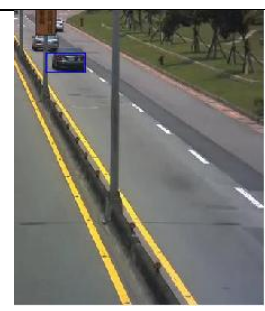

(b)

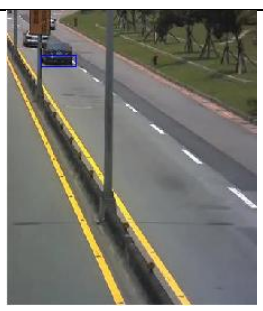

(c)

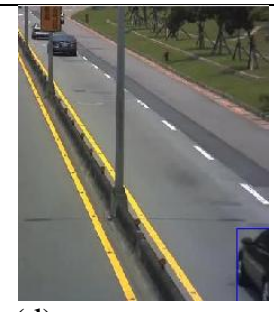

(d)

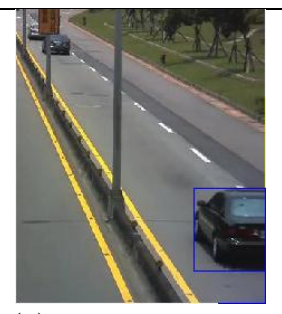

(e)

Fig. 4. Detection and tracking of vehicle by kalman filter for an image sequence. Please note that the given sequence is intermediate samples and not temporally interlinked with each other.

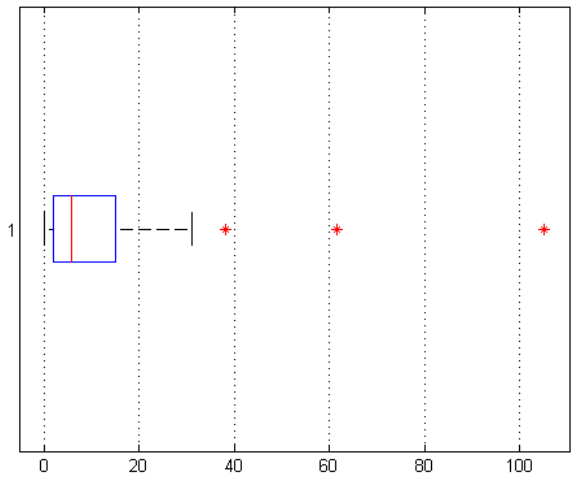

Fig 5 Box plot to describe the statistical relationship of the error between the detected and the actual posture of the vehicle

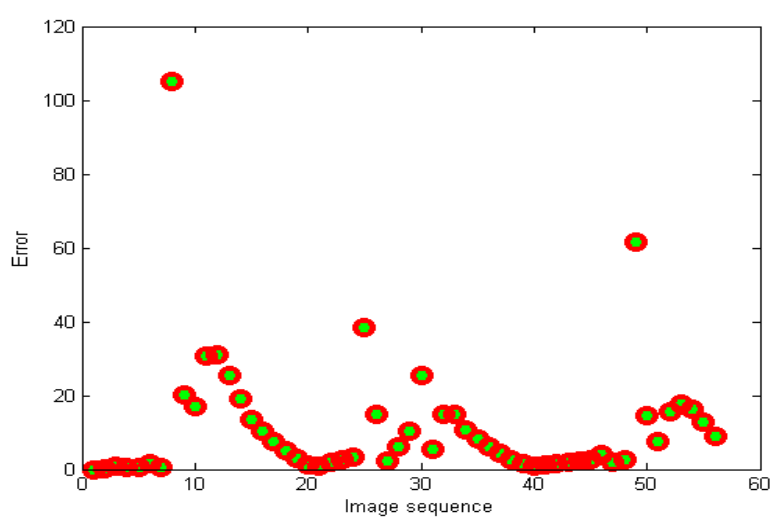

Fig 6 Instantaneous error between the detected and the actual posture of the vehicle

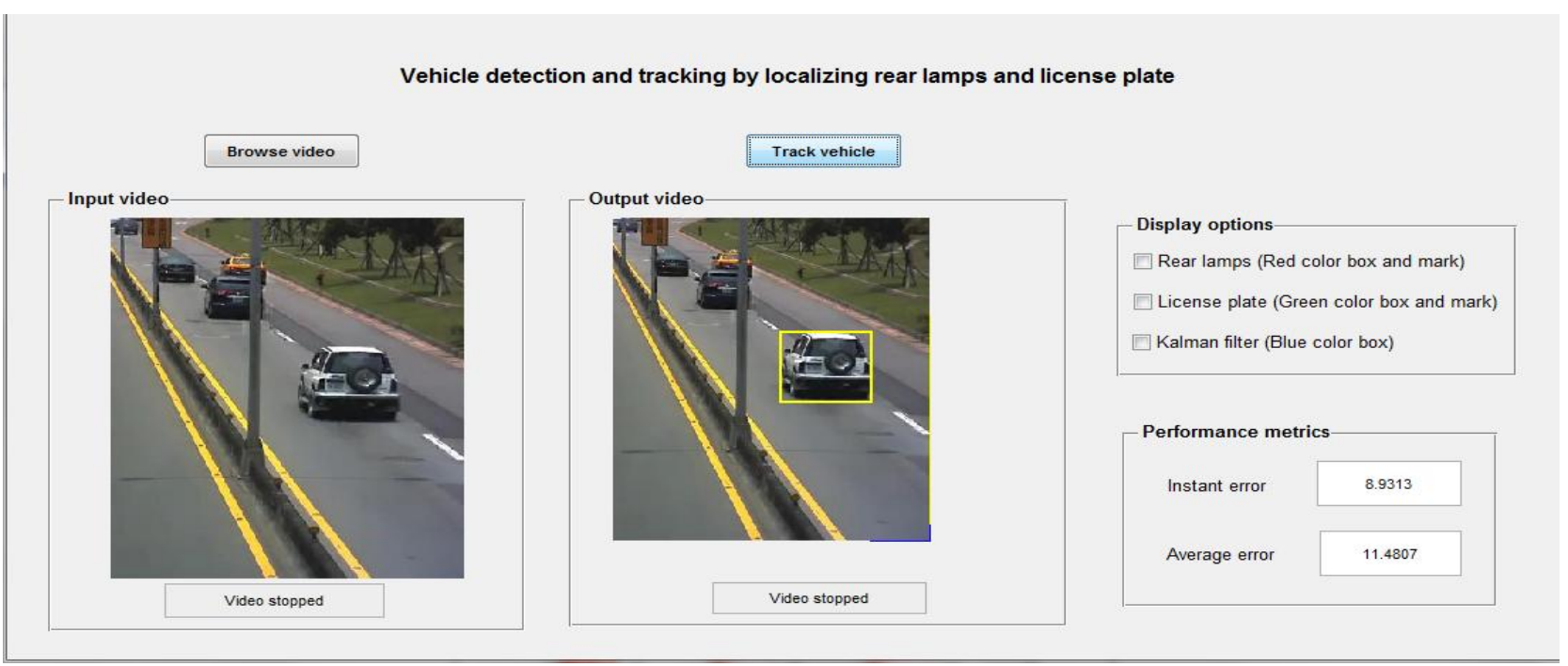

Fig 7 User interface of the proposed methodology developed in MATLAB 


\subsection{User Interface}

A user interface is developed in MATLAB for working on the methodology. The developed interface is illustrated in figure 7. The interface has a button, named as "Browse video" to browse and load input video, and an execution button, named "Track vehicle", to track vehicles in the loaded video.

The corresponding videos are played just below the buttons, whereas the status of the videos (whether it is being played or stopped) is displayed just below the video. The interface has three "Display options" to display the centroids of detected rear lamps and license plate as well as its approximated bounding region. Moreover, there is an option to display the kalman filter output also. The performance metrics are instantaneously updated in the "Instant error" box and the average of the instant errors are displayed in the "average error" box. The interface is developed for simple execution of the methodology. It shall be subjected to further enhancements so that the user can access the methodology intensely.

\section{CONCLUSION}

This paper has introduced a methodology to detect and track vehicles based on the location of rear lamps and license plate. Rear lamps and license plate of vehicles have been determined in a dedicated color space model to improve the precision. Kalman filter has been used to estimate the motion object as well as to ensure the performance of the rear lamp detection process and license plate detection process. The spatial relationship between the locations of the rear lamps and the license plate has been used to construct the markov model and hence the vehicle has been detected and tracked throughout the video. The experimental study on the methodology has revealed its reduced deviation between the actual vehicle and the tracked vehicle. The methodology shall be considered for further enhancement so that it can be applied in many real world applications, especially in intelligent transportation systems, surveillance, etc.

\section{REFERENCES}

[1]. Bin Tian; Ye Li; Bo Li; Ding Wen, "Rear-View Vehicle Detection and Tracking by Combining Multiple Parts for Complex Urban Surveillance", IEEE Transactions on Intelligent Transportation Systems, Volume: 15, Issue: 2, pp. 597 - 606, 2014

[2]. Chung-Ming Kuo; Chaur-Heh Hsieh; Yue-Dar Jou; Hsieh-Cheng Lin; Po-Chiang Lu, "Motion estimation for video compression using Kalman filtering", IEEE Transactions on Broadcasting, Vol. 42, No. 2 pp. 110 - 116, 1996

[3]. Jaemin Kim; Woods, J.W., "3-D Kalman filter for image motion estimation", IEEE Transactions on Image Processing, Vol. 7, No. 1, pp. 42 - 52, 1998, DOI: 10.1109/83.650849

[4]. Chung-Ming Kuo; Shu-Chiang Chung; Po-Yi Shih, "Kalman filtering based rate-constrained motion estimation for very low bit rate video coding", IEEE Transactions on
Circuits and Systems for Video Technology, Vol. 16, No. 1, pp. 3-18, 2006

[5]. W. Jones, "Keeping cars from crashing," IEEE Spectrum,September, pp. 40-45, 2001

[6]. Z. Sun, G. Bebis, and R. Miller, "On-road vehicle detection using gabor filters and support vector machines," in Proc. IEEE Conf. Digital Signal Process., 2002, vol. 2, pp. 1019-1022.

[7]. M. Betke, E. Haritaglu and L. Davis, "Multiple vehicle detection and tracking in hard real time," IEEE Intelligent Vehicles Symposium, pp. 351-356, 1996.

[8]. M. Cheon,W. Lee, C. Yoon, and M. Park, "Vision-based vehicle detection system with consideration of the detecting location," IEEE Trans. Intell. Transp. Syst., vol. 13, no. 3, pp. 1243-1252, Sep. 2012.

[9]. Mithun, N.C.; Rashid, N.U.; Rahman, S.M.M., "Detection and Classification of Vehicles From Video Using Multiple Time-Spatial Images", IEEE Transactions on Intelligent Transportation Systems, Vol. 13, No. 3, 2012

[10]. Michalopoulos, P.G., "Vehicle detection video through image processing: the Autoscope system", IEEE Transactions on Vehicular Technology, Vol. 40, No. 1, pp. 21 - 29, 1991

[11]. Yang Wang, "Real-Time Moving Vehicle Detection With Cast Shadow Removal in Video Based on Conditional Random Field", IEEE Transactions on Circuits and Systems for Video Technology, Vol. 19, No. 3, pp. 437 - 441, 2009

[12]. Kalman, R. E. (1960). "A New Approach to Linear Filtering and Prediction Problems". Journal of Basic Engineering 82: 35. doi:10.1115/1.3662552.

[13]. Fruhwirth, R. (1987). "Application of Kalman filtering to track and vertex fitting". Nucl. Instrum. Meth. A262 (2-3): 444-450.

Bibcode:1987NIMPA.262..444F. doi:10.1016/0168-9002(87)90887-4.

[14]. Leslie Pack Kaelbling; Michael L Littman \& Anthony R Cassandra (1998). "Planning and acting in partially observable stochastic domains" Artificial Intelligence (Elsevier) 101 (1-2): 99-134

[15]. Bin Tian; Ye Li; Bo Li; Ding Wen, "Rear-View Vehicle Detection and Tracking by Combining Multiple Parts for Complex Urban Surveillance", IEEE Transactions on Intelligent Transportation Systems, Volume: 15, Issue: 2, pp. $597-606,2014$

[16]. W. Jones, "Keeping cars from crashing," IEEE Spectrum, September, pp. 40-45, 2001

[17]. Z. Sun, G. Bebis, and R. Miller, "On-road vehicle detection using gabor filters and support vector machines," in Proc. IEEE Conf. Digital Signal Process., 2002, vol. 2, pp. 1019-1022.

[18]. M. Betke, E. Haritaglu and L. Davis, "Multiple vehicle detection and tracking in hard real time", IEEE Intelligent Vehicles Symposium, pp. 351-356, 1996.

[19]. M. Cheon,W. Lee, C. Yoon, and M. Park, "Visionbased vehicle detection system with consideration of the detecting location," IEEE Trans. Intell. Transp. Syst., vol. 13, no. 3, pp. 1243-1252, Sep. 2012.

[20]. Mithun, N.C.; Rashid, N.U.; Rahman, S.M.M., "Detection and Classification of Vehicles From Video Using Multiple Time-Spatial Images", IEEE Transactions on Intelligent Transportation Systems, Vol. 13, No. 3, 2012 
[21]. Michalopoulos, P.G., "Vehicle detection video through image processing: the Autoscope system”, IEEE Transactions on Vehicular Technology, Vol. 40, No. 1, pp. 21 - 29, 1991

[22]. Yang Wang, "Real-Time Moving Vehicle Detection With Cast Shadow Removal in Video Based on Conditional Random Field", IEEE Transactions on Circuits and Systems for Video Technology, Vol. 19, No. 3, pp. 437 - 441, 2009

[23]. Hu, Weiming, Tieniu Tan, Liang Wang, and Steve Maybank. "A survey on visual surveillance of object motion and behaviors." Systems, Man, and Cybernetics, Part C: Applications and Reviews, IEEE Transactions on 34, no. 3 (2004): 334-352. [2] Buch, Norbert, Sergio A. Velastin, and James Orwell. "A review of computer vision techniques for the analysis of urban traffic." Intelligent Transportation Systems, IEEE Transactions on 12, no. 3 (2011): 920-939.

[24]. Buch, Norbert, Sergio A. Velastin, and James Orwell. "A review of computer vision techniques for the analysis of urban traffic." Intelligent Transportation Systems, IEEE Transactions on 12, no. 3 (2011): 920-939

[25]. Barnich, Olivier, and Marc Van Droogenbroeck. "ViBe: A universal background subtraction algorithm for video sequences." Image Processing, IEEE Transactions on 20, no. 6 (2011): 1709-1724.

[26]. Elgammal, Ahmed, David Harwood, and Larry Davis. "Non-parametric model for background subtraction." In Computer Vision-ECCV 2000, pp. 751-767. Springer Berlin Heidelberg, 2000.

[27]. Toyama, Kentaro, John Krumm, Barry Brumitt, and Brian Meyers. "Wallflower: Principles and practice of background maintenance." In Computer Vision, 1999. The Proceedings of the Seventh IEEE International Conference on, vol. 1, pp. 255-261. IEEE, 1999.

[28]. Panda, Deepak Kumar. "Motion detection, object classification and tracking for visual surveillance application." PhD dissertation, 2012.

[29]. Haritaoglu, Ismail, David Harwood, and Larry S. Davis. "W4: real-time surveillance of people and their activities." Pattern Analysis and Machine Intelligence, IEEE Transactions on 22, no. 8 (2000): 809-830.

[30]. Antoine Manzanera and Julien C. Richefeu. A new motion detection algorithm based on [Sigma]-[Delta] background estimation. Pattern Recognition Letters, 28(3):320-328, February 2007.

[31]. Manuel Vargas. et al."An Enhanced Background Estimation Algorithm for Vehicle Detection in Urban Traffic Scenes", IEEE Transactions On Vehicular Technology, Vol. 59, No. 8, October 2010

[32]. Nicholas A., Iphigenia K.\& Chris T. Kiranoudis, "A background subtraction algorithm for detecting and tracking vehicles. ELSEVIER Expert Systems with Applications 38 (2011) 1619-1631 